\title{
P07-07. Non-progressive paediatric HIV infection is associated with virus attenuation and increase in $C D 8+$ specific $T$ cell responses over time
}

\author{
JG Prado*1, A Prendergast ${ }^{1}$, C Thobakgale ${ }^{2}$, C Juarez ${ }^{1}$, A Tudor-Williams ${ }^{3}$, \\ T Ndung' $\mathbf{u}^{2}$, BD Walker ${ }^{4}$ and P Goulder ${ }^{1}$
}

\author{
Address: ${ }^{1}$ University of Oxford, Fundacion irsiCaixa, Badalona, Spain, ${ }^{2}$ Univeristy of KwaZulu-Natal Durban, Durban, South Africa, ${ }^{3}$ Department \\ of Paediatrics/Imperial College, London, UK and ${ }^{4}$ Ragon institute/Massachusetts General Hospital, Boston, MA, USA \\ * Corresponding author
}

from AIDS Vaccine 2009

Paris, France. 19-22 October 2009

Published: 22 October 2009

Retrovirology 2009, 6(Suppl 3):PI05 doi:10.II86/I742-4690-6-S3-PI05

This abstract is available from: http://www.retrovirology.com/content/6/S3/PI05

(c) 2009 Prado et al; licensee BioMed Central Ltd.

\section{Background}

The majority of Human Immunodeficiency Virus (HIV)infected infants in the developing world progress to AIDS or death within the first year of life. Although a small minority become slow-progressors, the determinants of slow-progression remain to be elucidated. In this context the interactions between the characteristics of the maternal virus transmitted and the infants' adaptive immune responses are likely to be important. We undertook this study to elucidate the contribution of viral and host factors to HIV progression in perinatally-infected infants.

\section{Methods}

We studied a subgroup of 8 infants: 3 Progressors (P) and 5 Slow-Progressors (SP) with C-clade HIV infection, from a well-characterized sub-Saharan infant cohort. 19 longitudinal virus isolates were extracted from plasma samples by CD44-positive selection. We measured replicative capacity in CD8-depleted PBMCs. Virus were sequenced and tropism determined in U87 cells. Presence of mutations at Gag HLA-B*57/5801 epitopes evaluated. Optimal HIV CD8+ specific responses were measured by INFgamma ELISPOT and HLA class I types.

\section{Results}

We found that SP infants carry HIV isolates with low replicative capacity compared to virus from typical P. Furthermore, the attenuated viral phenotype can be linked to specific Gag mutational patterns of the transmitted virus and not to differences in coreceptor use or presence Nef polymorphisms between groups. We observed an increase in the breadth and magnitude of HIV CD8+ T-cell responses during the follow-up period in SP infants. Additionally, those infants were more likely to have protective class I HLA alleles. Meanwhile, P showed limited CD8+ Tcell responses over time and more often shared maternal HLA class I molecules, resulting in a transmitted virus preadapted to potential T-cell responses in the child.

\section{Conclusion}

These data demonstrate the contribution of both viral attenuation and CD8+ T-cell responses in paediatric HIV control. 